\title{
Experiments on the Role of the Question Under Discussion for Ambiguity Resolution and Implicature Computation in Adults
}

\author{
Arjen Zondervan, Luisa Meroni and Andrea Gualmini \\ Utrecht Institute of Linguistics (UiL-OTS)
}

\section{Introduction}

The starting point of this paper is an experiment by Hulsey et al. (2004), in which they show that children are sensitive to the context when they are interpreting a sentence containing a scope ambiguity. The contextual property they take into account is the question that was raised in the context, usually referred to as the Question Under Discussion (QUD, Roberts 1996). In this paper we will examine whether adults also rely on the QUD when they encounter a scope ambiguity. Also, we will investigate whether the effect of the QUD reaches further than scope ambiguities, into other domains where two interpretations seem to be competing with each other. To answer this question we will look at sentences containing a scalar implicature (SI)-trigger. But before we present the experiments, we need to look at the background against which the experiment of Hulsey et al. was set up.

Over the last ten years, there has been a lively discussion in the acquisition literature about the way young children deal with scope ambiguities involving a quantifier and negation. The discussion was triggered by a finding of an experiment reported in Musolino (1998), testing children's interpretation of by now well-known sentences like (1), that contain a quantifier and negation:

Every horse didn't jump over the fence.

In adult English, this sentence has two readings. The surface scope (SS) reading, in which the universal quantifier takes scope over the negation, can be paraphrased as for every horse it holds that it did not jump over the fence; and the inverse scope (IS) reading, where the negation scopes over the universal quantifier: not every horse jumped over the fence. Confronted with a story in which two out of three horses jumped over the fence, adult speakers of English judged sentence (1) true. This is expected, as one of the two possible readings of the sentence (the inverse scope reading) makes it true. However, in Musolino's experiment, 5-year-old children only accepted the sentence in $10 \%$ of the cases. Musolino concluded that children can only compute surface scope, and do not

Thanks to Marc Garellek, Katherine McCurdy, Anne Johnson, Mark Pollard, Michelle StAmour and Eva Sokol for help with data collection. This work was supported by a SSHRC Standard Research Grant, a VIDI grant from the Netherlands Organization for Scientific Research (NWO) to Andrea Gualmini and a Marie Curie Fellowship to Luisa Meroni. 
have access to the inverse scope reading, an idea called The Observation of Isomorphism.

Gualmini (2004) conducted a slightly adapted version of Musolino's experiment, with sentences containing negation and the existential quantifier some, e.g. (2):

The troll didn't deliver some pizzas.

As young children have not yet acquired the PPI-properties of some, this sentence has the same potential to be ambiguous for them as (1). It is ambiguous between the surface scope reading: it is not the case that the troll delivered some (=any) pizzas, and the inverse scope reading: some pizzas were such that they were not delivered. Isomorphism predicts only the first reading to be available. Gualmini presented the sentence following a story about a pizza-delivery troll, in which it was made very explicit that the troll was supposed to deliver all the pizzas. Eventually, the troll failed at this because he lost some pizzas on the way. In this situation, the surface scope reading of the sentence, which according to Isomorphism is the only one available to children, is false. However, in $90 \%$ of the cases, children accepted the target sentence. Apparently, they did have access to the inverse scope reading which made the sentence true. Gualmini concluded that the expectation of the troll delivering all the pizzas facilitated the inverse scope reading. This expectation raised the question (the QUD) whether the troll succeeded in delivering all the pizzas. In light of this question, the inverse scope reading, in which there were some pizzas he did not deliver, becomes much more salient than in a context in which such a question was not raised, as in the experiment by Musolino. Thus, the Observation of Isomorphism arguably arose because the contexts of Musolino's experiment were not appropriate to yield inverse scope interpretations.

However, a third player entered the field. Another factor that is well known to play a crucial role in choosing between two readings of one sentence is the Principle of Charity: Language users prefer the reading of the sentence that makes the sentence true. Proponents of Isomorphism now argued that although both readings are in principle available to the child, surface scope is their default interpretation, and inverse scope only comes about by costly reanalysis, for which children often lack the processing capacity (Musolino \& Lidz 2003). However, in cases where the surface scope reading is false, and the inverse scope reading is true, there is competition between (avoiding) this processing difficulty and the Principle of Charity. This explains why children sometimes manage to select inverse scope if that is the reading that makes the sentence true, as in the experiment by Gualmini.

To tease apart the claims of the QUD-driven account of Gualmini's results and the Isomorphism-by-Default claim of Musolino \& Lidz, Hulsey et al. (2004) set up an experiment in which the surface scope reading was the one that made the sentence true, but the QUD-account would predict inverse scope. They used the passive version of the sentences of Gualmini (2004), in which the existential quantifier and negation have switched places on the surface structure, e.g. (3). 


\section{(3) Some pizzas were not delivered.}

Just like (2), this sentence is potentially ambiguous for children. However, in the same situation in which the troll delivered two out of four pizzas, it is the surface scope reading that is true instead of the inverse scope reading. Therefore, there is no competition between Charity and processing load, and Isomorphism predicts children to accept this sentence. The QUD-approach also predicts acceptance here. If the QUD is Did the troll deliver all the pizzas?, the surface scope reading (that some pizzas were such that they were not delivered) directly addresses the QUD. In line with both views, children accepted the target sentence in $88 \%$ of the cases. As this condition did not tease the two views apart, Hulsey et al. introduced a second condition, in which the verb was changed to its contextual alternative, see (4):

Some pizzas were not lost.

The QUD-approach makes a different prediction in this condition. As the story is still the same, the QUD is still whether the troll delivered all the pizzas. The surface scope reading, that at least some pizzas were such that they were not lost, does not entail an answer to this question. The inverse scope reading, however, does address the QUD: it is not the case that some (=any) pizzas were lost is equivalent to all pizzas were delivered. The QUD-account therefore predicts the acceptance rate to drop, as inverse scope should be selected more often. On the other hand, Isomorphism predicts no difference: surface scope still makes the sentence true so there is no competition between Charity and processing load. According to Isomorphism, the acceptance rate of (3) and (4) should be comparable. The results presented by Hulsey et al. were that sentences like (4) were accepted in only $43 \%$ of the cases. Even though inverse scope made the sentence false, thereby going against Charity, in $57 \%$ of the cases children still selected this interpretation, as it was the only reading that provided an answer to the QUD.

Hulsey et al. conclude the QUD-view made better predictions than Isomorphism, and they propose the Question Answer Requirement (QAR):

\section{The Question Answer Requirement}

The selected interpretation of an ambiguous sentence, whether true of false, is required to be a good answer to the Question Under Discussion.

(A good answer is an interpretation that at least entails an answer to the QUD.)

The goal of the current work is to find out whether this sensitivity to the QUD also holds for adults. Therefore, we conducted two experiments, one designed to replicate the effect found by Hulsey et al. for adult speakers, and one investigating whether the QUD plays a comparable role in a different phenomenon, that of scalar implicatures. 


\section{Experiment 1}

\subsection{Setup and items}

The sentences that Hulsey et al. used are not ambiguous for adults (see Horn, 1989, p. 490), so we switched back to the universal quantifier. Target sentences were of the form in (6), with the two readings given below: ${ }^{1}$

(6) I think all the pizzas were not delivered.

SS: For all the pizzas it holds that they were not delivered.

IS: Not all the pizzas were delivered.

We used the same type of story that was used in Gualmini (2004) and Hulsey et al. (2004): the pizza delivery troll managed to deliver two out of four pizzas. Relative to this story, the surface scope reading is false, and the inverse scope reading is true. So acceptance of the target sentence indicated inverse scope.

As we wanted to investigate the role of the QUD and, in particular, to scrutinize the claim that the effect was due to the QUD, we included an explicit QUD in the experiment, while everything else was kept constant over conditions. Instead of asking the puppet to describe what happened, we asked the puppet to answer an explicit question. This question was manipulated over the two conditions. An example of an item of Condition 1 is given in (7):

Condition 1

Story summary: Pizza delivery troll delivers two out four pizzas, loses two.

Explicit QUD: "Were all the pizzas delivered?"

Target: "I think all the pizzas were not delivered."

According to the QAR, only the reading that at least entails an answer to the QUD will be selected. The QUD is questioning whether all the pizzas were delivered, or not all pizzas were delivered. This corresponds to the partition of the logical space, which can be conceived as the set of possible worlds, given in (8):

(8)

All pizzas were delivered.

Not all pizzas were delivered.

The surface scope reading is: for all the pizzas it holds that they were not delivered. This asymmetrically entails that not all pizzas were delivered, so it answers the QUD by entailment. The inverse scope reading, not all the pizzas were delivered is equivalent to one of the blocks in the partition in (8), so it

\footnotetext{
${ }^{1}$ The answers of the puppet started with I think in order to make them sound more natural as answers to a yes/no-question.
} 
directly answers the QUD. As both readings are good answers to the QUD, the QAR predicts no preference for one over the other, and we expect that participants accept the target sentence because of Charity. In Condition 2 however, the explicit QUD was changed to a question of the type exemplified in (9), with its partition in (10).

Condition 2

Explicit QUD: "Were some pizzas delivered?"

(10)

Some (and possibly all) pizzas were delivered.

No pizzas were delivered.

The story and the target sentence were the same as in Condition 1. The surface scope reading is equivalent to the lower block of the partition in (10), so it directly answers the QUD. The inverse scope reading however, only tells us that not all pizzas were delivered, leaving open the QUD whether some pizzas or no pizzas at all were delivered. As this reading does not entail an answer to the QUD, while surface scope does, we expect participants to select surface scope here. Remember that this is the reading that makes the sentence false, so we expect the acceptance rate to drop compared to Condition 1.

\subsection{Design, participants and procedure}

To avoid the risk of carry-over between the two conditions, we chose a betweensubjects design, in which 15 adult native speakers of English were assigned to List 1, containing only items of Condition 1, and 16 speakers to List 2, with only Condition 2-items. We adopted the Truth Value Judgment Task paradigm (TVJT, Crain \& Thornton, 1998). The stories were acted out with props in front of the participant, and after the story the experimenter posed the QUD to the puppet. The puppet then answered with the target sentence, and participants were asked to judge whether the puppet's answer was right or wrong. Every participant gave judgments on 4 test trials, interspersed with fillers.

\subsection{Results and Discussion}

In Condition 1, subjects overwhelmingly accepted the target sentence: In 98\% of the cases $(59 / 60)$ participants judged the sentence 'right'. This indicates they accessed the inverse scope interpretation that made the sentence true. In Condition 2 however, acceptance dropped to $23 \%(15 / 64)$, indicating here that in the majority of the cases participants selected the surface scope reading of the target sentence. This is in accordance with the predictions made by the QAR, as only surface scope provided an answer to the QUD in this condition. The difference between the two conditions was significant $\left(\chi^{2}=72.188, \mathrm{p}<0.0005\right)$. As the QUD is the only factor that is manipulated between the two conditions, we can 
conclude that the QUD has a significant effect on scope ambiguity resolution not only for children, but for adults as well.

The predictions of the QAR combined with Charity seem to be borne out in this experiment. We predicted that in Condition 1, participants would accept the sentence because of the Principle of Charity, and the results confirmed that prediction. However, the $77 \%$ rejection of the target sentence in Condition 2 goes against Charity. There was a reading of the sentence that made the sentence true (surface scope), of which we know it is accessible to adults, but still the reading that made the sentence false (inverse scope) was selected more often. We could hypothesize that in these cases, there is competition between the QAR and Charity: participants want the sentence to answer the QUD, but they also want the sentence to be true. In Condition 1 this competition was absent: Both readings were good answers to the QUD, so Charity governed the participants' behavior.

However, there is an alternative approach we could take. The two conditions that relied on Charity (i.e., Hulsey et al.'s Condition 1 in (11) and Condition 1 of the current experiment in (12)) are repeated below:

implicit QUD: Were all pizzas delivered?

target sentence: Some pizzas were not delivered.

explicit QUD: Were all pizzas delivered?

target sentence: All the pizzas were not delivered.

In (11), the selection of SS was explained by Charity, and so is the selection of IS in (12). However, these were the two readings that were a direct answer to the QUD, the readings that mapped onto one of the blocks of the partition of the QUD. Their competitors, IS in (11) and SS in (12), only asymmetrically entailed an answer. We could therefore propose a stricter version of the QAR, that does not just require entailment, but requires the reading to be (equivalent to) one of the alternatives denoted by the question. This way, Charity is no longer needed to explain the preferences found: the stricter version of the QAR can account for the whole observed paradigm. However, the current experiment does not distinguish between these two hypotheses, so we will leave this here as a suggestion for further research here.

Irrespective of the issue whether we need Charity or a stricter version of the QAR to explain the acceptance in Condition 1, we can conclude from Experiment 1 that adults, just like children, are guided by the QUD in selecting the reading of a scopally ambiguous sentence. In the next section, we will consider another domain in which effects of QUD have been reported: scalar implicatures.

\section{Scalar Implicatures and QUD}

Scalar Implicatures (SIs), typically exemplified by the inference from some to not all and from or to not and, are widely studied as they are reasonably robust 
pragmatic inferences. However, they are known to disappear, or get reversed, in certain environments. A number of factors that are responsible for this disappearance have been described in the literature, almost all of them being sentence-level properties. For instance, SI-rates have been shown to decrease in downwards entailing environments, such as the antecedent of a conditional, or embedded under negation (see Chierchia et al. 2001). However, little is known about which properties at the contextual level block SIs. Zondervan (2007) and (in press-a) proposes a contextual property that affects SI-calculation: the QUD. He provides experimental evidence that more SIs are drawn for the scalar or in the (Dutch equivalents of the) dialogue in (13) than in (14), although in both cases or is part of the same sentence.

$$
\begin{aligned}
& \text { A: "What did Harry bring?" } \\
& \text { B: "Harry brought bread or chips." } \\
& \text { A: "Who brought bread or chips?" } \\
& \text { B: "Harry brought bread or chips." }
\end{aligned}
$$

According to Zondervan, the QUD determines which part of the sentence gets focus. This is a property which has been proposed by Van Kuppevelt (1996) and Van Rooij \& Schulz (2004) to affect SIs. ${ }^{2}$ Van Rooij \& Schulz introduce a silent exhaustivity operator (exh), which applies to the interpretation of a sentence and gives it the SI. However, they claim that exh only applies to the constituent of the sentence that has focus, so SIs only arise in focused constituents. What is crucial about the accounts of Van Kuppevelt and Van Rooij \& Schulz, is that they claim the interpretation of declarative sentences depends on the question the sentence is an answer to. For instance, if the sentence John is sick is uttered in response to the question Who is sick?, the sentence is usually interpreted to mean that only John is sick, while as an answer to What's the matter with John? there might be more people in the relevant domain that are sick. This question that affects the meaning of the sentence might be explicitly given, or - which is often the case - it is implicit in the context. This is how the sentence-level property of focus becomes a contextual property: which part of the sentence gets focus depends on the contextual question it is an answer to, the QUD. If the scalar term is in this focused part, as in (13), the SI will arise. If, however, another part of the sentence is questioned by the QUD, as in (14), where the subject is questioned and or is in the object, the SI will not arise. Zondervan (2007) and (in press-a) presents experimental support for this hypothesis. He reports significantly higher SI-rates for items like (13), than for (14).

To demonstrate that the QUD is a truly contextual property than can also be implicit, Zondervan conducted an experiment in which the QUD was not explicitly given, but had to be distilled from the story. In an example story of the first condition, four students were introduced, who were going for lunch in a

\footnotetext{
${ }^{2}$ Van Kuppevelt uses the term comment, which roughly corresponds to focus as defined by Jackendoff (1972): information assumed by the speaker not to be shared by the hearer.
} 
cafeteria. They get to the fruit section, which only had apples and pears. The story then considered the choice of the students one by one, e.g. John thought the apples and pears looked old, so he didn't take any. ${ }^{3}$ In the end, only one student took something, and she actually took an apple and a pear. Because the four students were considered one by one, and there was no contrast set for the object (there were no other types of fruit than apples and pears), the QUD that was triggered was about the subject: Who took an apple or a pear? The pre-recorded target sentence, given in (15) was played with focus intonation on the subject, corresponding to this QUD.

PAOLA took an apple or a pear from the fruit section.

Or was not in a focused constituent in this condition. In the corresponding story in the other condition, there was only one student going for lunch, Paola. However, now there were four types of fruit to choose from. The story considered the types of fruit one by one, saying of each of them if and why Paola did or did not take one, e.g. Bananas, Paola didn't like. So she didn't take one. The topicalization structure was chosen to emphasize the fact that the types of fruit were listed. In this condition there was no contrast set for the subject (no other students), but there was a contrast set for the object. The most salient QUD therefore was about the object: What (types of fruit) did Paola take? The pre-recorded target sentence was now played with focus intonation on the object, an apple or a pear.

Paola took AN APPLE OR A PEAR from the fruit section.

In this condition, or was in a focused constituent. Therefore Zondervan expected more SIs to arise in this condition than in the first condition. Indeed, a significant difference was found between the two conditions in a between-subjects study with 20 and 25 participants. In line with the predictions, more SIs ( $84 \%$ vs. 54\%) were drawn in the second condition, the condition in which or was in a focused constituent. This also shows that if the QUD is not explicitly given, but is implicit in the context, it has the same effect on SI-calculation.

To recap, Experiment 1 showed language users' sensitivity to the QUD in scope ambiguity resolution, and the studies by Zondervan showed effects of QUD on scalar implicatures. In the current work we wanted to bring the results of both studies together. One thing we wanted to find out was whether the effect on SIs that Zondervan found with wh-questions could also be found with the yes/noquestions we used in Experiment 1. In addition, we wanted to check whether the generalization we proposed based on the scope data, the QAR, would make the right predictions for scalar implicatures. Therefore we conducted another experiment.

\footnotetext{
${ }^{3}$ The use of or in the stories was avoided.
} 


\section{Experiment 2}

\subsection{Setup and items}

To investigate whether the results found by Zondervan could be extended to the type of QUDs we had been using in the scope experiment, and to see whether the QAR would make the right predictions here too, we designed an experiment testing for SIs with our yes/no QUDs of Experiment 1. We presented participants with the same type of story as in Experiment 1, but this time the troll actually managed to deliver all four pizzas. The target sentence contained the scalar term some, which is normally associated with the SI not all. An example of a target sentence is given in (17), with the two readings (with and without scalar implicature) given below it: ${ }^{4}$

(17) Some pizzas were delivered.

+ SI: Some but not all pizzas were delivered.

- SI: Some and possibly all pizzas were delivered.

In the story in which the troll delivered all the pizzas, the SI would make the sentence false. If no SI is drawn, the sentence should be considered true. Acceptance of the target sentence would therefore indicate that the SI was not drawn. The critical manipulation of this experiment was varying the explicit QUD over conditions, just like in Experiment 1. The QUDs were of the same type as those used in Experiment 1. An example of an item of Condition 1 is given in (18):
Condition 1
Explicit QUD: "Were some pizzas delivered?"
target: "Some pizzas were delivered."

As we assume that SIs do not arise in questions (see Noveck et al. 2002, but compare Zondervan, in press-b), we expect the QUD in (18) to denote the partition in (19):

(19)

Some and possibly all pizzas were delivered.

No pizzas were delivered.

If participants calculate the SI for the target sentence, it provides an answer to the QUD, as some but not all pizzas were delivered entails some and possibly all

\footnotetext{
${ }^{4}$ We present the two alternatives (with and without SI) in the same way as we presented the two readings of an ambiguous sentence. This is just for ease of reference, we are not making the claim that a sentence containing a scalar term is ambiguous between its implicature reading and its no implicature reading in the same way a scopally ambiguous sentence is. In scope ambiguities, there are two possible LFs, while traditionally, implicatures are considered to apply after the LF of the sentence has been computed.
} 
pizzas were delivered. But also without the SI, the target sentence is a good answer to the QUD, it corresponds to the top block in (19). The original version of the QAR therefore predicts that participants will accept the sentence because of Charity. The stricter version of the QAR presented in section 2.3 also predicts acceptance, as the sentence without SI is a direct answer. ${ }^{5}$

In Condition 2, we changed the quantifier in the QUD to all. An example is given in (20), and the partition is given in (21):

\section{Condition 2}

Explicit QUD: "Were all pizzas delivered?" target: "Some pizzas were delivered."

(21)

All pizzas were delivered.

Not all pizzas were delivered.

In this condition, the QUD is only answered if the SI is calculated: Some but not all pizzas were delivered (the sentence with SI) entails that not all pizzas were delivered, but some and possibly all pizzas were delivered tells us nothing about this question. The QAR therefore predicts that participants will draw the SI here, and acceptance of the sentence will drop.

\subsection{Design, participants and procedure}

A between-subjects design was used, with 14 adult native speakers of English in one group and 14 in the other. The paradigm we used was again the TVJT. Every participant gave judgments on 4 trials of the same condition, mixed with fillers.

\subsection{Results and Discussion}

In Condition 1, participants accepted the target sentence $93 \%$ of the times $(52 / 56)$, indicating they accessed the reading without the implicature, as QAR and Charity predicted. On Condition 2, the acceptance rate was 57\% (32/56), indicating that in $43 \%$ of the cases, participants drew the SI. This is a significant effect of condition $\left(\chi^{2}=19.05, p<0.0005, t=4.75, p<0.0005\right)$. These results support the original QAR: More SIs were calculated in Condition 2, as there the interpretation with SI provided an answer to the QUD, while the reading without SI did not. ${ }^{6}$ The results

\footnotetext{
${ }^{5}$ A number of authors discuss similar examples, claiming the SI is absent in this case. For example Carston (1998) cites example (i) by Green (1995):

(i) B: Are some of your friends Buddhist? A: Yes, some of them are.

${ }^{6}$ Notice that the results do not support the stricter version of the QAR, because the reading with SI in Condition 2 only answers the QUD by asymmetrically entailing an answer, it is not equivalent to one of the blocks in the partition. On the strict version of the SI, both readings in
} 
show that the type of QUDs we have seen to affect scope ambiguity resolution also have an effect on SI-calculation, in the way the QAR predicted.

One question that remains is how these results relate to the focus-based account of how the QUD affects SIs, that was put forward by Zondervan. Let us look at the predictions this account would make for this experiment. In Condition 1 , an affirmative answer is given to a yes/no question, by repeating the questioned statement as a declarative. There is no part of the answer that provides new information (information assumed by the speaker not to be shared by the hearer), so we could argue that this whole sentence is focus-less. ${ }^{78}$ If this is the case, the scalar term is also in a non-focused constituent, and Zondervan's focus hypothesis predicts the SI not to arise, which is indeed what we found. In Condition 2 however, there is a part of the answer that contains new information, as all in the question is replaced by some in the answer. So in this condition some is focused, and more SI are predicted to arise than in the non-focused Condition 1. This prediction is also confirmed by the data. We can conclude that in this experiment, the focus account of how the QUD affects interpretation makes the same predictions as the QAR, and therefore it is an alternative explanation of the data.

\section{Conclusion}

In this paper we set out to find experimental evidence that adult language users rely on the QUD when they are confronted with a sentence containing a scope ambiguity, and that this effect extends to other domains where there is competition between two meanings. Experiment 1 showed that for the same target sentence, different readings are preferred following different explicit QUDs. In the condition in which only one reading was an answer to the QUD, participants selected this reading, even though it made the sentence false. The QAR proposed by Hulsey et al. was shown to also hold for scope ambiguity resolution of adults. Experiment 2 built on work by Zondervan (2007, and in press-a), in which it is shown that the QUD of the context has a significant effect on the calculation of scalar implicatures. In Experiment 2, we replicated this effect with the yes/noQUDs that guided ambiguity resolution in Experiment 1. Again, the predictions of the QAR were borne out: participants calculated more implicatures when only the reading with implicature addressed the QUD. Taken together, the results of the two experiments show that one contextual property, that of QUD, can account for participants' preferences on two apparently distinct phenomena: scope ambiguities and scalar implicatures. This result is an important step in the process of understanding how context drives interpretation.

Condition 2 are not good answers to the QUD, so it would predict the no SI reading based on Charity.

${ }^{7}$ The only new information is an implicit 'yes', that is communicated through repeating the questioned statement as a declarative.

${ }^{8}$ But see Zondervan (in press-b) for a more detailed discussion about this assumption, based on the notions of topicalized and neutral yes/no-questions. 
The fact that both investigated domains are governed by a single contextual property, suggests that this property might be crucial in other domains too, and that the QUD might be a general property of how context affects interpretation.

\section{References}

Carston, R. (1998). Informativeness, relevance, and scalar implicature. In R. Carston, \& S. Uchida (Eds.), Relevance theory: applications and implications. Amsterdam: John Benjamins.

Chierchia, G., S. Crain, M.T. Guasti, A. Gualmini and L. Meroni (2001). The acquisition of disjunction: Evidence for a grammatical view of scalar implicatures. In A. H.-J. Do et al. (eds.), BUCLD 25 Proceedings, 157-168.

Crain, S. \& R. Thornton. (1998). Investigations in Universal Grammar: A Guide to Experiments on the Acquisition of Syntax and Semantics. Cambridge, Mass.: MIT Press.

Green, M. (1995) "Quantity, volubility, and some varieties of discourse." Linguistics and Philosophy 18, 83-112.

Gualmini, Andrea (2004) The Ups and Downs of Child Language, Outstanding Dissertations in Linguistics series, Routledge, New York, NY.

Horn, L. R., (1989). A natural history of negation. Chicago: University of Chicago Press.

Hulsey, S., Hacquard, V., Fox, D., and Gualmini, A., (2004). The QuestionAnswer requirement and scope assignment. In A. Csirmaz and A. Gualmini and A. Nevins (Eds.), Plato's problem: problems in language acquisition (pp. 71-90). MITWPL, Cambridge, Mass.

Jackendoff, R. (1972). Semantic Interpretation in Generative Grammar, Cambridge, Mass. MIT Press.

van Kuppevelt, J. (1996). Inferring from topics. Scalar implicatures as topic dependent inferences. Linguistics and Philosophy 19, 393-443.

Musolino, J. (1998). Universal Grammar and the Acquisition of Semantic Knowledge: an Experimental Investigation into the Acquisition of Quantifier-Negation Interaction in English. PhD Dissertation, University of Maryland, College Park.

Musolino, J., and Lidz, J., 2003. The Scope of Isomorphism: Turning Adults into Children. Language Acquisition, 11(4), 277-291.

Noveck, I., G. Chierchia, F. Chevaux, R. Guelminger and E. Sylvestre (2002) Linguistic-pragmatic factors in interpreting disjunctions. Thinking \& Reasoning, 8 (4), 297-326

Roberts, C. (1996). Information structure in discourse: Towards an integrated formal theory of pragmatics. Papers in Semantics, OSU Working Papers in Linguistics, Vol 49. Department of Linguistics, the Ohio State University, Columbus.

van Rooij, R. \& K. Schulz (2004). Exhaustive interpretation of complex sentences. Journal of Logic, Laguage and Information 13(4): 491-519. 
Zondervan, A. (2007). Effects of question under discussion and focus on scalar implicatures. Proceedings of the Fifth Semantics in the Netherlands Day, 39-52.

Zondervan, A. (in press-a). Experiments on QUD and focus as a contextual constraint on scalar implicature calculation. In: U. Sauerland and K. Yatsushiro (eds.) From Experiment to Theory, Proceedings of Experimental Pragmatics 2007, Palgrave Macmillan.

Zondervan, A. (in press-b) The role of QUD and focus on the scalar implicature of most. To appear in: Meibauer, J. and M. Steinbach, (eds.) Proceedings of the workshop on Experimental Semantics/Pragmatics at the DGfS 2008. 